\title{
Three-dimensional structure of a mutant E.coli aspartate aminotransferase with increased enzymic activity
}

\author{
Joachim Jäger ${ }^{1,2}$, Richard A.Pauptit ${ }^{1,3}$, Ursula Sauder ${ }^{1}$ \\ and Johan N.Jansonius ${ }^{1,4}$

\begin{abstract}
${ }^{1}$ Abteilung Strukturbiologie, Biozentrum, Universıtät Basel, Klingelbergstrasse 70, CH-4056 Basel, Switzerland, '2Department of Molecular Biophysıcs and Biochemistry, Howard Hughes Medıcal Institute, Yale University, 266 Whitney Avenue, New Haven, CT 06511, USA and ${ }^{3}$ ICI Pharmaceuticals, Macclesfield SK 10 4TG, UK
\end{abstract} \\ ${ }^{4}$ To whom correspondence should be addressed
}

The aspartate and tyrosine aminotransferases from Escherichia coli have $43 \%$ sequence identity and nearly identical active sites. Both are equally good enzymes for dicarboxylate substrates, but the latter transaminates aromatic amino acids 1000 times faster. In an attempt to discover the critical residues for this differential substrate specificity, the aspartate aminotransferase mutant V39L has recently been prepared. It showed improved $k_{\mathrm{cat}} / K_{\mathrm{m}}$ values for aspartate, glutamate and tyrosine and the corresponding oxo acids, mainly due to two to ten times lower $K_{\mathrm{m}}$ values. For example, the $\boldsymbol{K}_{\mathrm{m}}$ values of V39L (wild type) for Asp and Glu are 0.12 (1.0) and $0.85(2.7) \mathrm{mM}$ respectively. The mutant was co-crystallized with $30 \mathrm{mM}$ maleate from both polyethylene glycol and ammonium sulfate. Both structures were solved and refined to $R$-factors of 0.22 and 0.20 at 2.85 and $2.5 \AA$ resolution respectively. They bear strong resemblance to the closed structure of the wild type enzyme complexed with maleate. The unexpected feature is that, for the first time, the closed form was produced in crystals grown from ammonium sulfate. It is concluded that the mutation has shifted the conformational equilibrium towards the closed form, which leads to generally reduced substrate $K_{\mathrm{m}}$ s.

Key words: aspartate aminotransferase/conformational change/ enzyme specificity/point mutation/X-ray structure

\section{Introduction}

Aspartate aminotransferase (AspAT; EC 2.6.1.1) was the first representative of the large family of vitamin $\mathbf{B}_{6}$-dependent enzymes involved in nitrogen metabolism to be isolated in pure form (Jenkins et al., 1959). Its properties have been thoroughly investigated (Braunstein, 1973; Christen and Metzler, 1985; Torchinsky, 1986; Jansonius and Vincent, 1987). AspATs are $\alpha_{2}$-dimers with $\sim 400$ amino acid residues and one molecule of the co-factor pyridoxal-5'-phosphate (PLP) per chain. It employs a ping - pong bi-bi mechanism (Cleland, 1963) to catalyse the reversible amino group transfer reaction L-aspartate +2 -oxoglutarate $\leftrightharpoons$ oxaloacetate $+\mathrm{L}$-glutamate in which the enzyme shuttles between a PLP and a pyridoxamine-5'-phosphate (PMP) form. Vertebrates contain two AspAT isozymes, a cytosolic form (cAspAT) with 411 or 412 residues per chain and a mitochondrial form (mAspAT) with 401 residues per chain. The best characterized bacterial AspAT is that of Escherichia coli (eAspAT), the product of the aspC gene, with 396 residues per chain. The many known sequences can be uniquely aligned due to a high level of similarity (Christen and Metzler, 1985; Mehta et al., 1989) and it has become common praxis to use the residue numbering of the first determined and longest sequence of pig cytosolic AspAT (Ovchinnikov et al., 1973; Doonan et al., 1975) throughout. The crystal structures of the cytosolic isozyme from pig and chicken and of the chicken mitochondrial enzyme were determined more than 10 years ago (reviewed in Christen and Metzler, 1985; Torchinsky, 1986; Jansonius and Vincent, 1987). Inhibitor binding studies revealed conformational changes involving both the co-factor and the protein matrix and provided the basis for proposals of the stereochemistry of the catalytic reaction (Kirsch et al., 1984; Arnone et al., 1985a; Jansonius and Vincent, 1987).

Interest in the catalytic mechanism of AspAT was further enhanced when it became possible to determine the roles of specific active site residues by kinetic studies of site-directed mutants. Malcolm and Kirsch (1985), Toney and Kirsch (1987), Kuramitsu et al. (1987), Cronin and Kirsch (1988) and Inoue et al. (1989) are some early examples of these studies. Because of the ease of expression of large quantities, eAspAT mutants were used in these studies in most cases. Naturally, this triggered $\mathrm{X}$-ray diffraction studies of the $E$.coli enzyme. Its structure was solved independently by Kamitori $\mathrm{et} \mathrm{al.} \mathrm{(1987,} \mathrm{1988,} \mathrm{1990),} \mathrm{by}$ Smith et al. $(1986,1989)$ and by Jäger $e t$ al. (1989). The fold and the active site structure of eAspAT were found to correspond closely to those of the vertebrate enzymes.

The two identical shared active sites of AspAT have evolved to bind dicarboxylic acid substrates, mainly through hydrogenbonded ion pairs with active site arginines. The $\alpha$-carboxylate of the substrate interacts with Arg386 and its $\omega$-carboxylate with Arg292 of the neighbouring subunit. Although significant catalytic activity towards other amino acids would a priori seem improbable, aromatic amino acids are (albeit poor) substrates for AspAT (Shrawder and Martinez-Carrion, 1972; Mavrides and Christen, 1978; Kuramitsu et al., 1990), with an efficiency increasing in the order of cAspAT $\leq$ mAspAT < eAspAT. Even more intriguing are the catalytic properties of E.coli tyrosine aminotransferase (eTyrAT, EC 2.6.1.5, the product of the tyrB gene) in comparison with eAspAT. Sequence alignment shows that these enzymes have $43 \%$ sequence identity, with all catalytic and substrate binding residues of AspATs being conserved in eTyrAT (Fotheringham et al., 1986). Thus, it is not surprising that the latter is an equally efficient enzyme for dicarboxylic acid substrates as the former (Powell and Morrison, 1978). The puzzling feature is, how does eTyrAT manage to be three to four orders of magnitude more effective on aromatic amino acids than eAspAT (Hayashi et al., 1993; Köhler et al., 1994) despite the fact that all residues known to play a role in co-factor or substrate binding and in catalysis in eAspAT are conserved in eTyrAT (Fotheringham et al., 1986; Seville et al., 1988; Jäger et al., 1992)?

This intriguing phenomenon has, for several years, been the subject of investigations in the laboratory of K.Kirschner (Biozentrum, Basel). Seville et al. (1988) built models of both eAspAT and eTyrAT using the refined structure of a complex between mAspAT and maleate in space group $\mathrm{C}_{222}$ as a 
template (Jansonius et al., 1985; Jansonius and Vincent, 1987; Picot et al., 1991). The enzyme in this complex adopts the closed conformation. Seville et al. (1988) demonstrated that plausible models of eAspAT and eTyrAT could be built in this way, essentially without changing the $\alpha$-carbon skeleton of mAspAT. Moreover, the substrate L-tyrosine could, as an 'external' aldimine to PLP, be oriented in a productive conformation into the active site of the closed eTyrAT model structure. This was a further indication of the feasibility of the eTyrAT model. On the basis of these models and prior to the existence of a crystal structure of eAspAT, the residues of mAspAT, eAspAT and eTyrAT at identical positions in and around the active site were compared. This analysis enabled the design of mutant eAspATs and eTyrATs with hybrid active sites (Köhler, 1990; Köhler et al., 1994). In this way attempts to find the key residue(s) responsible for activity towards tyrosine and hydroxyphenylpyruvate (HOPP) were made.

One promising candidate for such an exercise was residue 39 which is Ala in mAspAT, Val in eAspAT and Leu in eTyrAT. Its bulkiness parallels activity towards aromatic amino and oxo acid substrates. Accordingly, the V39L mutant eAspAT was produced and characterized, in the hope that it would have enhanced activity towards tyrosine. A kinetic analysis revealed unexpected, but very interesting properties. Instead of being an improved tyrosine aminotransferase, the mutant eAspAT was generally a 'better' enzyme. The $k_{\text {cat }}$ values for the various substrates were essentially identical to wild type eAspAT, but the $K_{\mathrm{m}}$ values were reduced by factors between two and ten, with corresponding increases in $k_{\mathrm{cal}} / K_{\mathrm{m}}$ (which expresses substrate specificity; Fersht, 1985). For example, $K_{\mathrm{m}}$ and $k_{\text {cat }}$ for the substrates Asp and Glu (as determined for the half-reaction with HOPP as oxo acid substrate) for V39L (in parentheses wild type) are $K_{\mathrm{m}}^{(\text {Asp) }}=0.12(1.0) \mathrm{mM}, k_{\text {cat }}^{(\text {Asp })}=85(68) / \mathrm{s}, K_{\mathrm{m}}^{\text {(Glu) }}=0.85$ (2.7) $\mathrm{mM}$ and $k_{\text {cat }}^{\text {(Glu) }}=243(220) / \mathrm{s}$ (Köhler et al., 1994). The same mutant was also studied by Hayashi et al. (1991) with qualitatively similar results.

In order to investigate the structural basis for the curious effect exerted by a single additional $\mathrm{CH}_{2}$-group, in a residue not in direct contact with either substrate or co-factor (Jansonius and Vincent, 1987; Seville et al., 1988; Jäger, 1991), the V39L mutant eAspAT was crystallized in collaboration with Kirschner and colleagues (Jäger et al., 1989) and the crystal structure of a complex with the non-covalent inhibitor maleate was determined using crystals of space group $\mathrm{P} 21$ grown with either polyethylene glycol of average mol. wt 4000 (PEG) or ammonium sulfate as precipitant. We describe here the results of that study and show that the, for five out of six substrates (Köhler et al., 1994), lowered $K_{\mathrm{m}}$ values that characterize this mutant can, in all likelihood, be attributed to a shift of the open-closed conformational equilibrium towards the closed structure.

\section{Materials and methods}

\section{Cloning, mutagenesis and purification}

The cloning, mutagenesis and expression of the aspC gene of E.coli encoding eAspAT have been described by Fotheringham et al. (1986). The construction, protein purification and biochemical characterization of the V39L mutant enzyme was carried out by E.Köhler and K.Kirschner [for details see Köhler (1991); Köhler et al. (1994)]. In all cases eAspAT was produced in the strain $E$. coli $\mathrm{HW} 857\left[\mathrm{~F}^{-} \Delta(\operatorname{asp} C) \mathrm{Kan}^{R} \mathrm{tyr} B / \mathrm{Tn} 10\right]$, kindly provided by I.Fotheringham and M.Edwards.
Table I. Summary of crystallization and data collection statistics

\begin{tabular}{|c|c|c|}
\hline Code name & V39L-PEG & V39L-AS \\
\hline Ligand & $30 \mathrm{mM}$ maleate & $30 \mathrm{mM}$ maleate \\
\hline Precipitant & $\begin{array}{l}31 \%(w / v) P^{2} G_{4000} \\
\text { pH } 75\end{array}$ & $\begin{array}{l}48 \% \text { saturated } \mathrm{AS}^{\mathrm{a}} \\
\text { pH } 7.5\end{array}$ \\
\hline Crystal size (mm) & $1.8 \times 0.5 \times 0.5$ & $0.8 \times 0.7 \times 04$ \\
\hline Detector & XENTRONICS & FAST \\
\hline Image width $\Omega\left({ }^{\circ}\right)$ & 0.25 & 0.15 \\
\hline Exposure time (s) & 200 & 60 \\
\hline Cell axes $(a, b, c)(\dot{\mathrm{A}})$ & 86.479 .989 .8 & 86.479 .889 .7 \\
\hline Cell angle $\beta\left(^{\circ}\right)$ & 118.6 & 118.7 \\
\hline$V_{\mathrm{m}}\left(\dot{\mathrm{A}}^{3} / \mathrm{Da}\right)^{\mathrm{b}}$ & 3.11 & 3.10 \\
\hline$N^{\mathrm{c}}$ & 2 & 2 \\
\hline Resolution limit $(\dot{\mathrm{A}})$ & 2.75 & 2.5 \\
\hline Number of measurements & 61788 & 96603 \\
\hline Number of unique data & 26470 & 35641 \\
\hline$R_{\mathrm{sym}}(I)(\%)^{\mathrm{d}}$ & 6.2 & 6.6 \\
\hline $\begin{array}{l}\text { Completeness to resolution } \\
\text { limit }(\%)\end{array}$ & 92.9 & 96.2 \\
\hline
\end{tabular}

${ }^{\mathrm{a} A S}$, ammonum sulfate.

${ }^{\text {b}}$ Ratio of volume of asymmetric unit to mol. wt (Matthews, 1968)

${ }^{\mathrm{C}}$ Number of subunits per asymmetric unit.

${ }^{\mathrm{d}} R_{\mathrm{sym}}=\frac{\Sigma_{h k l} \cdot \Sigma_{j}\left|I_{j, h k l}-<I_{h k l}\right\rangle \mid}{\Sigma_{h k l} \Sigma_{j} I_{j, h k l}}$

where $I_{J, h k l}$ is the intensity from measurement $j$ of reflection $h k l$ and $\left\langle I_{h k l}\right\rangle$ the mean value of $j=1, \ldots, N$ observations of symmetry equivalent reflections $h k l$.

\section{Crystallization, data collection and processing}

Crystals were grown at $\mathrm{pH} 7.5$ by the hanging drop method (McPherson, 1982). The drops $(20 \mu \mathrm{l})$ contained $12.5 \mathrm{mg} / \mathrm{ml}$ protein, $30 \mathrm{mM}$ maleate and as precipitants either $12.5 \%(\mathrm{w} / \mathrm{v})$ PEG or $24 \%$ saturated ammonium sulfate in a $20 \mathrm{mM}$ sodium phosphate buffer, $\mathrm{pH} 7.5$, with, as further additions, $1 \mathrm{mM}$ each of 2-oxoglutarate, EDTA, dithiothreitol, as well as $10 \mu \mathrm{M}$ PLP and $0.02 \%$ sodium azide. The wells contained $1 \mathrm{ml}$ of either $23-25 \%$ PEG or $45-48 \%$ saturated ammonium sulfate in

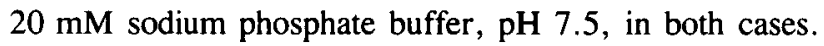

The crystals belong to the monoclinic space group $\mathrm{P} 2_{1}$ with unit cell parameters of typically $a=86.2, b=79.8, c=89.5 \AA$ and $\beta=118.7^{\circ}$. Intensity data sets were collected on both XENTRONICS and FAST area detector systems using graphite monochromated $\mathrm{CuK}_{\alpha}$ radiation. Intensity data collected on the XENTRONICS system were processed and reduced using the XENGEN software (Howard et al., 1987). The data from the FAST area detector system were processed using the MADNES software (Messerschmidt and Pflugrath, 1987). Symmetry equivalent reflections were averaged and merged giving final $R_{\text {sym }}$-values of 6.2 and $6.6 \%$ for V39L-PEG and V39L-AS respectively. Characteristics of the crystal forms and data collection statistics are summarized in Table I.

\section{Structure determination}

The V39L-PEG structure was solved by molecular replacement, using a modified chicken mAspAT structure (Eichele et al., 1979; Ford et al., 1980) in which the side chains were exchanged to conform to the eAspAT wild type sequence as a trial model (Seville et al., 1988). Three regions where deletions occurred within the eAspAT sequence compared with that of mAspAT were found to be within surface loops of the molecule. The rotational search was performed using the CCP4 programs ALMN (by E.Dodson) and POLARRFN (by W.Kabsch), which 
are based on the fast rotation function (Crowther, 1972). The dimeric trial model was positioned with its longest dimension approximately parallel to the body diagonal of a Pl unit cell with axial lengths of $a=b=c=100 \AA$ and angles of $90^{\circ}$. Structure factors were calculated between 14 and $3 \AA$, assuming an overall temperature factor of $25 \AA^{2}$. The integration radius in Patterson space was chosen to be $30 \AA$ ( $20 \AA$ for POLARRFN). The translational search was performed using the program TFSGEN (Tickle, 1985), which makes use of the $T_{2}$-translation function of Crowther and Blow (1967). Observed data between 15 and $3 \dot{A}$ were included. After the initial structure solution, rigid body refinement (CORELS, Sussman et al., 1977) was used to further improve the V39L-PEG model. The second structure (V39L-AS) was interpreted by inspection of $\left(F_{\mathrm{obs}}-F_{\text {calc }}\right)$, $\alpha_{\text {calc }}$ and $\left(2 F_{\text {obs }}-F_{\text {calc }}\right), \alpha_{\text {calc }}$ Fourier maps, in which the calculated structure factors were taken from the refined V39L-PEG structure.

\section{Structure improvement}

Interactive rebuilding. A Sim-weighted $\left(2 F_{\text {obs }}-F_{\text {calc }}\right), \alpha_{\text {calc }}$ electron density map for V39L-PEG was computed using the calculated phases and structure factor amplitudes derived from the rigid-body refined model. To reduce the bias towards the model structure this electron density calculation was limited to small portions of the molecule (typically 5-8\%), for which the contribution of calculated phases and structure factor amplitudes were omitted. Using a local procedure (by J.P.Priestle, Basel) these partial maps were merged to cover the molecule. In addition to this 'omit' map, $\sigma_{\mathrm{a}}$-weighted $\left(2 F_{\text {obs }}-F_{\text {calc }}\right), \alpha_{\text {calc }}(\operatorname{Read}, 1986)$ and $\left(3 F_{\text {obs }}-2 F_{\text {calc }}\right), \alpha_{\text {calc }}$ electron density difference maps were calculated for simultaneous usage. The dimer was rebuilt on an interactive Evans and Sutherland PS330 graphic display system using FRODO software (Jones, 1985), extended by P.R.Evans (MRC Cambridge, UK).

Least-squares refinement. Restrained least-squares refinement was carried out using the program package TNT (Tronrud et al., 1987). The weighting between the contributions from the crystallographic and stereochemical terms was carefully adjusted prior to positional refinement cycles by running test cycles using the steepest descent method. No stereochemical restraints towards deviation of torsional angles from ideal staggered conformations were used during refinement, since the rotational energy barriers between preferred side chain orientations are low.

Molecular dynamics refinement. Details of the different molecular dynamics refinement steps are listed in Table II. After determination of the ideal weights $W_{A}$ (stage 1 in Table II) the V39L-PEG model was submitted to an energy minimization procedure (XPLOR), including 8.0-3.0 $\AA$ data, to relieve structural stress. Two parallel runs of molecular dynamics simulations using the simulated annealing technique were applied to the model:

(i) dynamics simulation for $1.2 \mathrm{ps}$ at $2000 \mathrm{~K}$ and a subsequent $300 \mathrm{fs}$ fast cooling stage, including diffraction data between 8.0 and $3.0 \dot{A}$ (stage $3 \mathrm{a}$ in Table II);

(ii) molecular dynamics simulation for 1.7 ps following the slow cooling protocol, i.e. temperature bath coupled dynamics from $2000 \mathrm{~K}$ to $300 \mathrm{~K}$ including diffraction data between 8.0 and $2.85 \AA$ (stage $3 b$ in Table II).

The final stage (stages $4 a$ and $b$ in Table II) comprised 120 steps of energy minimization.
Table II. Steps and parameters used in V39L-eAspAT simulated annealing refinement

\begin{tabular}{|c|c|c|}
\hline \multicolumn{2}{|c|}{ Stage } & \multirow{2}{*}{$\begin{array}{l}\text { Description } \\
\text { Determination of weight } W_{A}: W_{A}=420000 \mathrm{kcal} / \mathrm{mol}\end{array}$} \\
\hline 1 & CHECK & \\
\hline 2 & PREPARE & $\begin{array}{l}160 \text { conjugate gradient steps energy minimization } \\
\mathrm{C}_{\alpha} \text {-restraints at } 20 \mathrm{kcal} /\left(\mathrm{mol} \dot{A}^{2}\right), \mathrm{d} F=0.05 \dot{A}\end{array}$ \\
\hline 3(a) & $\begin{array}{l}\text { EQUILIBRATION } \\
\text { and } \\
\text { FAST COOLING }\end{array}$ & $\begin{array}{l}1.2 \text { ps molecular dynamics, } T=2000 \mathrm{~K} \text {, } \\
10.0-3.0 \dot{A} \text {. integratuon step } 1 \mathrm{fs} \text {, velocity scaling } \\
\text { every } 25 \mathrm{fs}, \mathrm{d} F=0.2 \dot{A}, \text { followed by } 0.3 \mathrm{ps} \\
\text { molecular dynamics, } \mathrm{T}=300 \mathrm{~K}, 10.0-3.0 \dot{\mathrm{A}} \text {, } \\
\text { integration step } 1 \mathrm{fs} \text {, velocity scaling every } 25 \mathrm{fs} \text {, } \\
\mathrm{d} F=0.2 \dot{\mathrm{A}} \text {, followed by }\end{array}$ \\
\hline ) & $S$ & $\begin{array}{l}1.7 \text { ps temperature bath coupled molecular } \\
\text { dynamics, } T=2000-300 \mathrm{~K}, 10.0-2.85 \AA \\
\text { integration step } 0.5 \mathrm{fs}, W_{A}=390000 \mathrm{kcal} / \mathrm{mol} \text {, } \\
\mathrm{d} F=0.2 \AA\end{array}$ \\
\hline 4(a) & FINAL & $\begin{array}{l}120 \text { conjugate gradient steps energy minimization, } \\
10.0-3.0 \AA, \mathrm{d} F=0.05 \AA\end{array}$ \\
\hline 4(b) & $\mathrm{AL}$ & $\begin{array}{l}120 \text { conjugate gradient steps energy minimization, } \\
10.0-2.85 \dot{A}, \mathrm{~d} F=0.05 \dot{\AA}, \\
W_{A}=370000 \mathrm{kcal} / \mathrm{mol}\end{array}$ \\
\hline
\end{tabular}

\section{Results}

Structure solution and improvement

Different parameter combinations were tried in the rotational search applied to the V39L-PEG data. The best signal to noise ratio for the peaks found in the rotation function was obtained using a Patterson integration radius of 5-30 $\AA$ with data between 15 and $3 \AA$ included. The rotation matrix corresponding to the highest peak in the rotation function was applied to the model (the next highest peak had a height of $32 \%$ of the maximum rotation function value) and a translational search was performed using the program TFSGEN. The highest peak was found to be $11 \sigma$ above the mean (next highest peak $3.5 \sigma$ ). This gave a model for which an $R$-factor of 0.528 was calculated for the resolution range of $15-3 \AA$. An orthogonal translation vector of $(x, y, z$ $=22,0,11 \AA$ ) was applied to the model. The $y$ component of the translation vector is arbitrary in space group $P 2_{1}$. A 2-D $R$-factor search (along $x$ and $z$ ) in steps of $0.1 \AA$ around the translation function solution lowered the $R$-factor by $1 \%$ $(x, y, z=22.1,0,10.9 \AA)$. Using the program CORELS (Sussman et al., 1977), the overall orientation, position and the dimer structure of V39L-PEG eAspAT were improved by treating the two monomers separately during rigid body refinement. The centre of gravity coordinates did not change in this process, while rotations around the centre of the subunits were $<2^{\circ}$. After CORELS refinement, the model gave an $R$-factor of 0.488 for diffraction data between 15 and $3 \AA$ resolution. This preliminary model was improved by rebuilding the positions that did not fit well within the density of a Sim-weighted 'omit' map (see Materials and methods).

The atomic positions of the corrected model were subjected to 36 cycles of restrained least-squares refinement with the program package TNT (Tronrud et al., 1987), interspersed with manual rebuilding. This resulted in an $R$-factor of 0.305 for the data between 15.0 and $3.0 \AA$ resolution. The model was then entered into XPLOR refinement. One hundred and sixty steps of conjugate gradient energy minimization improved the overall $R$-factor to 0.252 for the data between 10.0 and $3.0 \dot{A}$ resolution, indicating that the errors in portions of the molecule had been beyond the convergence range of the TNT refinement procedure. The different simulated annealing refinement protocols (stages $3 \mathrm{a}$ 


\begin{tabular}{lcc}
\hline \multicolumn{3}{l}{ Table III. Summary of refinement parameters and statistics } \\
\hline Parameter & \multicolumn{2}{l}{ Structure } \\
\cline { 2 - 3 } & V39L-PEG & V39L-AS \\
\hline R-factor & 0.224 & 0.200 \\
Resolution range $(\dot{A})$ & $15-2.85$ & $15-2.5$ \\
Number of atoms & 6250 & 6392 \\
Protein & 6186 & 6186 \\
Solvent & $64^{\mathrm{a}}$ & 206 \\
R.m.s. deviations from ideal geometry & & \\
Bond lengths $(\dot{\mathrm{A}})$ & 0.015 & 0.015 \\
Bond angles $\left({ }^{\circ}\right)$ & 2.984 & 2.922 \\
Torsion angles $\left({ }^{\circ}\right)$ & 24.627 & 24.090 \\
Planar groups $\left({ }^{\circ}\right)$ & 1.838 & 1320 \\
Mean temperature factors $\left(\dot{A}^{2}\right)$ & & \\
Overall & 25.8 & 27.9 \\
Large domain & 22.4 & 23.9 \\
Small domain & 33.6 & 35.4 \\
Backbone atoms & 19.6 & 24.4 \\
Side chain atoms & 29.8 & 30.0 \\
Solvent molecules & - & 35.8 \\
\hline
\end{tabular}

${ }^{a}$ Due to limited resolution, manually inserted using graphics display system.

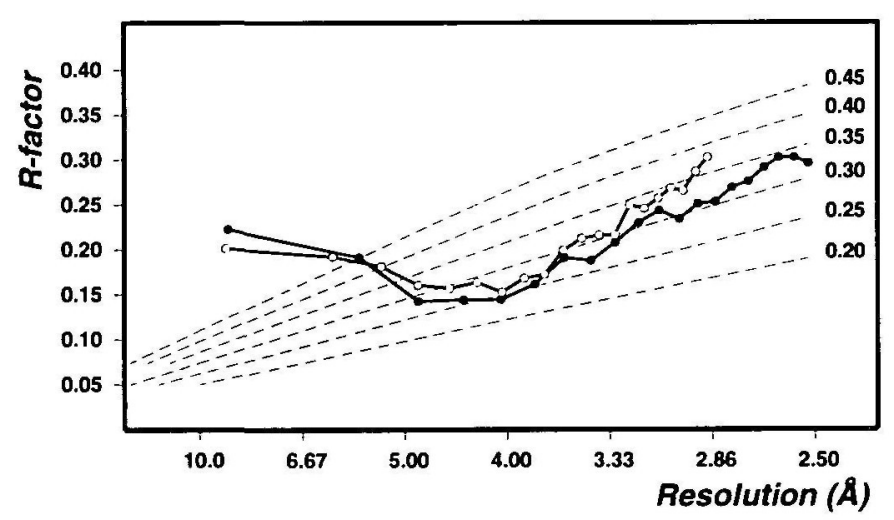

Fig. 1. Variation in $R$-factor as a function of resolution (Luzzati plot). The curves shown correspond to the V39L eAspAT-maleate complex structure (V39L-PEG, open circles) and to that of the V39L eAspAT-maleate complex crystallized from ammonium sulfate (V39L-AS, filled circles) The dashed lines represent the theoretical variation of the $R$-factor when coordinate errors between $\Delta r=0.2 \dot{\mathrm{A}}$ and $\Delta r=0.45 \dot{\mathrm{A}}$ are assumed (Luzzati, 1952).

and $\mathrm{b}$ in Table II) gave rise to structures of similar stereochemical quality, but the lowest $R$-factor was obtained with the slow cooling protocol. A total of 64 water molecules could be located in the final $\sigma_{\mathrm{a}}$ weighted $\left(2 F_{\text {obs }}-F_{\text {calc }}\right), \alpha_{\text {calc }}$ electron density map. Positions and temperature factors of the solvent molecules were not refined. Table III summarizes the refinement parameters and the final statistics of the V39L-PEG model.

The structure of the V39L-AS complex was solved by difference Fourier techniques taking calculated structure amplitudes and initial phases from the refined V39L-PEG model. The initial $R$-factor for V39L - AS was 0.255 at $2.5 \dot{A}$. A total of 206 solvent molecules could be inserted in $\left(F_{\text {obs }}-F_{\text {calc }}\right), \alpha_{\text {calc }}$ difference Fourier maps. Three hundred steps of energy minimization interspersed with 60 steps of individual temperature factor optimization lowered the $R$-factor to 0.20 including 35130 reflections between 10.0 and $2.5 \AA$ (see Table III).

\section{Quality of the models}

The $\left(F_{\text {obs }}-F_{\text {calc }}\right), \alpha_{\text {calc }}$ difference maps are essentially featureless and only few regions of the models have poorly defined

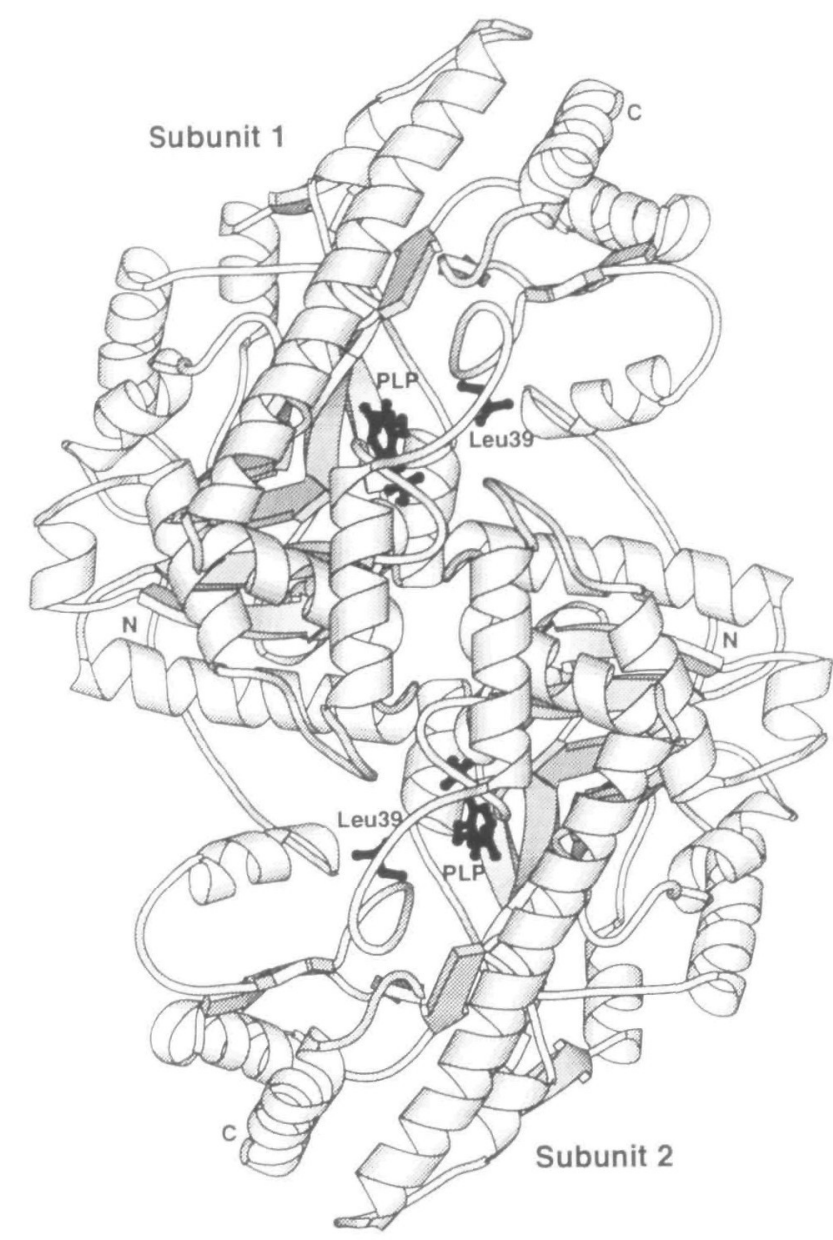

Fig. 2. Ribbon drawing (Krauls, 1991) of the overall structure of the V39L eAspAT dimer in the closed conformation. The view is along the molecular 2 -fold axis which is almost parallel to the $c^{*}$-axis. The long helix connecting the large and the small domains is kinked and serves as a pivot for opening and closing of the enzyme during the catalytic cycle. The active sites of the eAspAT dimer are located at the subunit interface and consist of residues from both large domains and one of the small domains. The cofactor PLP and the site of mutation (Val39 $\rightarrow$ Leu) are shown on both subunits

$\left(2 F_{\text {obs }}-F_{\text {calc }}\right), \alpha_{\text {calc }}$ electron density. These regions (residues $27-29$ and $344-347$ ) also exhibit very high temperature factors resulting in poor definition of side chain atomic positions. The least flexible polypeptide segments in each subunit, as indicated by the lowest temperature factors, occur in the large pleated sheet, including the co-factor binding residues in the active site region. The general quality of the models can be judged from the overall coordinate errors (Luzzati, 1952), by plotting the variation in the $R$-factor as a function of resolution or by the program SIGMAA (Read, 1986). Using SIGMAA, the r.m.s. coordinate errors for the maleate complexes were determined to be 0.45 and $0.42 \dot{A}$ respectively, which is somewhat higher than the errors derived from the Luzzati (1952) plot (Figure 1). Upon superposition of the backbone atoms $\left(\mathrm{N}, \mathrm{C}_{\alpha}, \mathrm{C}, \mathrm{O}\right) \operatorname{good}$ agreement is found for the two refined structures. The r.m.s. deviations are $<0.46 \dot{A}$, which is in the range of the overall coordinate errors. The r.m.s. deviation in the backbone atom positions between the initial model built from a coordinate set of chicken mAspAT (Seville et al., 1988) and the refined models of V39L eAspAT is $1.23 \AA$. The same differences $(1.24 \AA)$ are found if the V39L mutant structure (V39L-AS) is compared to the X-ray structure of chicken mAspAT, complexed with 
maleate (Jansonius and Vincent, 1987). Maximum deviations of $\sim 5 \AA$ occur in a loop around Met246 located at the surface of the molecule.

The $\phi$ and $\psi$ angles of all residues are in allowed areas of the Ramachandran plot with the following exceptions (the same in both subunits; the angles given refer to subunit 1): Phe377 $\left(-113^{\circ},-137^{\circ}\right.$, in a surface loop) and $\operatorname{Ser} 296\left(69^{\circ},-58^{\circ}\right.$, involved in substrate binding). Four non-glycine residues lie in the $\alpha_{\mathrm{L}}$-region: Lys $98\left(58^{\circ}, 49^{\circ}\right), \operatorname{His} 166\left(60^{\circ}, 58^{\circ}\right)$ and Cys 192 $\left(56^{\circ}, 71^{\circ}\right)$ which lie in surface loops, while Arg266 $\left(62^{\circ}, 83^{\circ}\right)$ is a co-factor binding residue. All these residues except Lys98 and Phe 377 have well defined side chain density.

\section{Discussion}

\section{Conformational state-comparison to wild type structure}

The overall structure of the eAspAT V39L mutant, which appears to be similar to those of the vertebrate AspAT isozymes (Ford et al., 1980; Arnone et al., 1985b; Borisov et al., 1985; Jansonius and Vincent, 1987) and to previously described eAspAT structures (Kamitori et al., 1988, 1990; Smith et al., 1989), is illustrated by a ribbon diagram (Kraulis, 1991) in Figure 2. Details of the fold and the domain structure of eAspAT have been described previously (Kamitori et al., 1988, 1990; Smith et al., 1989). Three structures of wild type eAspAT complexed with sulfate, maleate and 2-methylaspartate in space group $\mathrm{P} 2_{1}$ have been solved by X-ray crystallography (Jäger et al., 1994) using crystallization conditions that were also applied in the crystallization of the V39L mutant enzyme. Both wild type structures liganded with a substrate analogue, maleate or 2-methylaspartate, were found to be in the closed conformation, whereas the 'unliganded' structure (complexed with a sulfate ion) adopts a 'half-open' conformation (Smith et al., 1989). The closing of the structures involves a $6^{\circ}$ rotation of the small domain (residues 16-46 and 330-409) towards the centre of the rigid PLP binding domain. This causes a reorientation of the side chains of Asp15, Ile17, Val18, Ile37 and Arg386 in the active site such that tight contacts to the substrate are established, presumably prior to catalysis taking place.

Interestingly, the half-open form is not found in any of the crystal structures of the V39L mutant enzyme, although several crystallization experiments were designed to produce crystals of the half-open form, e.g. by crystallization from an ammonium sulfate buffer without maleate. UV crystal spectra and the $2.7 \AA$ electron density map in the active site region (data not shown) suggest that the binding pocket is occupied, presumably with 2-oxoglutarate, which was present in the crystallization buffer at a concentration of $\sim 1 \mathrm{mM}$. This result demonstrated a shift in the conformational equilibrium towards the closed structure, caused by the mutation V39L.

\section{The site of mutation}

In many aminotransferases, position 39 (pig cAspAT numbering) is occupied by a hydrophobic residue: alanine is found in all vertebrate mAspAT and cAspAT sequences while eAspAT has a valine and both eTyrAT and Saccharomyces cerevisiae histidinol phosphate aminotransferase have a leucine in this position. By contrast, an aspartate is found in E.coli histidinol phosphate aminotransferase and rat TyrAT (Mehta et al., 1989). Residue 39 is part of a cluster of hydrophobic residues ('hydrophobic patch') located at the active site entrance above the binding pocket. The hydrophobic patch comprises residues Ile17, Leu 18, Ala21, Ile37, Gly38, Tyr40, Thr47, Tyr263, Met359, Asn388, Ala390 and Asn69* and Tyr70* from the
Table IV. Nearest neighbours to Leu39 side chain atoms in structure V39L-AS

\begin{tabular}{|c|c|c|}
\hline Atom, & Atom, & $\begin{array}{l}\text { Distance } r_{i j}(\dot{A}) \text { in } \\
\text { subunit } S 1(\mathrm{~S} 2)\end{array}$ \\
\hline \multirow{5}{*}{ Leu39 $C_{\delta 1}$} & Ile37 $C_{\gamma 2}$ & $4.4(4.3)$ \\
\hline & Thr47 $\mathrm{C}_{\gamma}$ & $4.2(4.6)$ \\
\hline & $\operatorname{Asn} 69 * C_{\gamma}^{\prime}$ & $3.7(3.7)$ \\
\hline & $\operatorname{Asn} 69 * N_{\delta 2}^{\prime}$ & $2.7(2.8)$ \\
\hline & Asn69* $\mathrm{O}_{\delta 1}$ & $4.3(4.6)$ \\
\hline \multirow[t]{9}{*}{ Leu39 $C_{\delta 2}$} & Tyr263 $\mathrm{C}_{\gamma}$ & $3.8(3.6)$ \\
\hline & Tyr263 $C_{\delta 1}$ & $3.7(3.7)$ \\
\hline & Туг $263 \mathrm{C}_{\delta 2}$ & $3.7(3.7)$ \\
\hline & Tyr263 $\mathrm{C}_{\epsilon l}$ & $3.6(3.5)$ \\
\hline & $\operatorname{Tyr} 263 \mathrm{C}_{\zeta}$ & $3.6(3.6)$ \\
\hline & $\operatorname{Asn} 69 * N_{\delta 2}$ & $3.6(3.9)$ \\
\hline & Asn $69 * C_{\gamma}$ & $4.3(4.1)$ \\
\hline & Tyr $70 * \mathrm{C}_{\delta 1}$ & $3.9(4.1)$ \\
\hline & Tyr $70 * C_{\epsilon 1}$ & $4.1(4.0)$ \\
\hline \multirow[t]{4}{*}{ Leu39 $\mathrm{C}_{\gamma}$} & $\operatorname{Ile} 37 \mathrm{C}_{\gamma 2}$ & $4.4(4.4)$ \\
\hline & Ile37 C & $4.1(4.0)$ \\
\hline & Thr47 $\mathrm{C}_{\gamma}$ & $4.3(4.1)$ \\
\hline & $\operatorname{Asn} 69 * N_{\delta 2}$ & $4.0(3.9)$ \\
\hline Leu39 $C_{\beta}$ & Tyr263 $\mathrm{C}_{\zeta}$ & $4.5(4.6)$ \\
\hline
\end{tabular}

An asterisk indicates a residue from the second subunit.

adjacent subunit (an asterisk indicates a residue from the neighbouring subunit). The side chain of residue 39 is neither in direct contact with the substrate nor catalytically important in another way. Van der Waals contacts with neighbouring residues in the structure V39L - AS are listed in Table IV. It is interesting to note that in both conformational states of eAspAT valine (wild type) or leucine 39 (mutant) make contacts with residues $69 *$ and $70 *$ from the neighbouring subunit. The orientation of the Asn69* side chain adapts to the bulkiness of residue 39. Although a substitution of Val39 by Phe would increase the size of the side chain in this position it is doubtful whether this could be accommodated in the densely packed hydrophobic environment. In this respect it is anticipated that the bulkiness of Phe interferes with a complete closure of the active site, in accord with experimental evidence that the V39F mutant eAspAT is a somewhat less efficient enzyme than V39L and wild type eAspAT (Hayashi et al., 1991). Model building studies using the eAspAT V39L-maleate complex suggest that the reduced performance of the V39F enzyme mainly originates from steric effects of Phe39 on Ile37 and Ile 73* and that Phe39 should have little effect on the structural and functional role of Tyr70*, as was suggested by Hayashi et al. (1991). Ile37 is rotated out of the active site in the unliganded enzyme (Figure 4). Upon substrate or competitive inhibitor binding, it rotates back into the hydrophobic patch, thereby shielding the active site from bulk solvent. Phe39 disorganizes the packing of hydrophobic residues at the active site entrance. This results in an insufficient shielding of the active site as Ile37 is unable to rotate back into the hydrophobic patch and to fully obstruct the active site entrance in the closed conformation. An alanine residue is found in position 39 in all sequences of the vertebrate cytosolic and mitochondrial isozymes. The packing of Ala39 against neighbouring hydrophobic residues, e.g. Val37 and unfavourable contacts with charged residues, i.e. Arg41 and Glu69*, in the direct vicinity of the $\beta$-carbon atom of Ala39 preclude incorporation of larger hydrophobic residues in these isozymes. By contrast, Asn69* in 

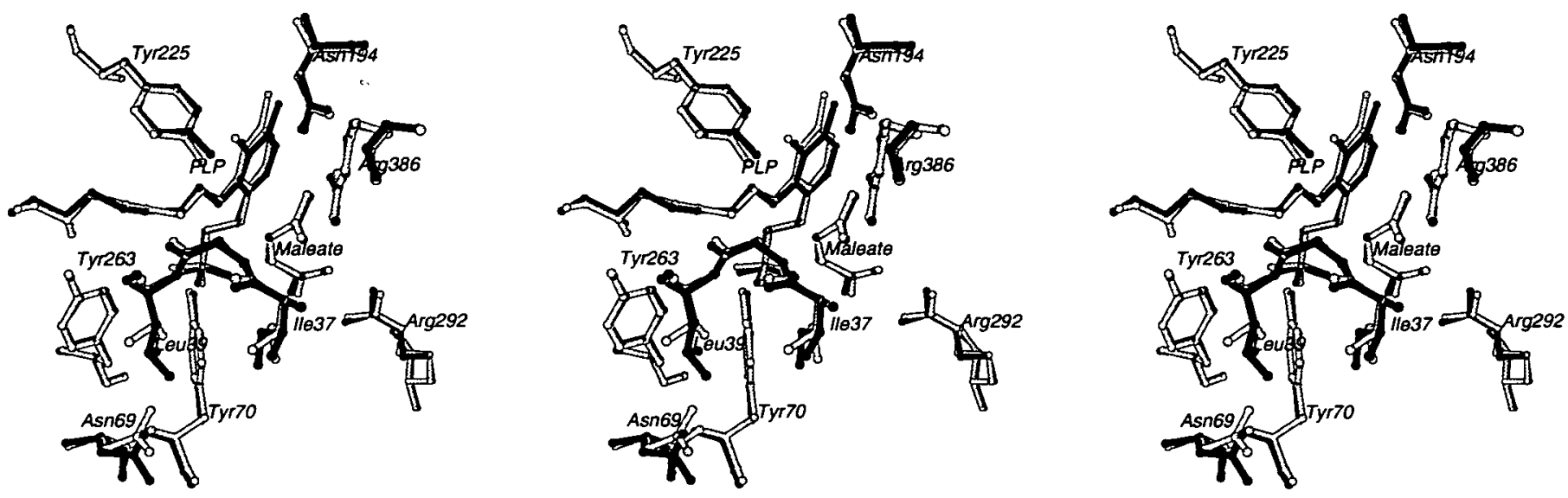

Fig. 3. Superposition of important active site residues in wild type (Jäger et al., 1994) and V39L eAspAT-maleate complexes. Residues Ile17 and Val18 (not shown), Ile37, Leu39 and Asn69* form a hydrophobic lid above the substrate binding pocket, shelding it from bulk solvent. The changes in the active site topology due to the mutation V39L are minute. Replacement of the Val39 side chain by that of Leu causes a shift in the position of the Asn69* amide group and a reorientation of the lle37 side chain to optimize its contacts with Leu39. The van der Waals interactions between $\mathrm{C}_{\delta 1}$ and $\mathrm{C}_{\delta 2}$ of Leu39 and residues Asn69*. Tyr 70* and Tyr263 are strengthening the subunit-subunit interactions and stabilizing the closed conformation. The wild type structure is depicted in grey.
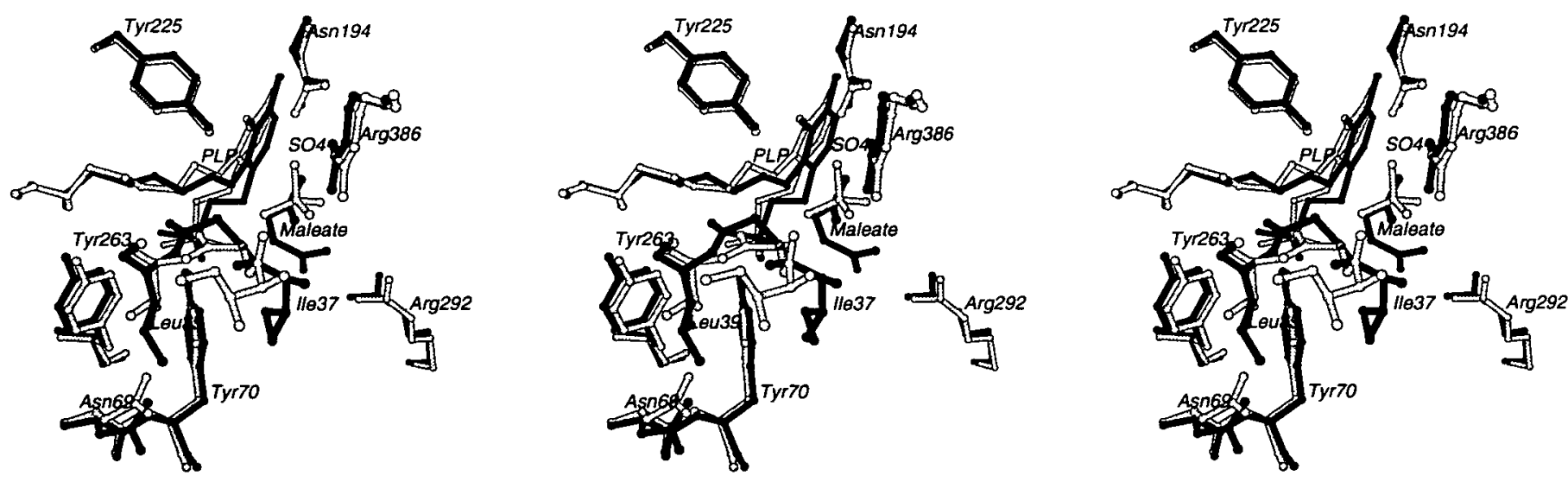

Fig. 4. Superposition of important active site residues in wild type (Jager et al., 1994) and V39L eAspAT-maleate complexes, both crystallized from ammonium sulfate. Maleate is found in the substrate binding pocket of the mutant enzyme only. A sulfate ion is found in the active site of the wild type enzyme that adopts the 'half open' conformation. Hence, sulfate is unable to trigger domain closure. In the closed structure (see Figure 3 ) He37 has nearly the same positions in the wild type and V39L eAspAT. Upon domain opening Ile 37 in the wild type eAspAT rotates by $110^{\circ}$. The mutant enzyme seems to bind substrate analogues and, presumably, substrates better than wild type eAspAT. The mutation brings about a shift in the conformational equalibrium from the open towards the closed conformation. The wild type structure is depicted in grey.

eAspAT and Leu69* in eTyrAT leave more space for bulkier side chains, such as valine and leucine, to form a tight hydrophobic lid above the active site.

\section{Active site topology-comparison to wild type structure}

The crystallization conditions with maleate as the substrate analogue and PEG as precipitant resulted in fully closed structures for both wild type and V39L mutant enzyme (V39L-PEG), as already predicted from UV absorption spectra of enzyme/inhibitor solutions and preliminary X-ray studies (Jäger et al., 1989). Changes in the orientation of side chains of active site residues and in the position of the substrate analogue arising from the replacement of valine by leucine are minute and within experimental error. The r.m.s. deviation in hydrogen bonding distances between maleate and its protein ligands in wild type and mutant eAspAT is $0.2 \dot{A}$ (Jäger, 1991). Close van der Waals interactions between the substrate analogue and hydrophobic side chains such as Ile17, Val18 and Ile37 are found in both structures. The side chain amide group of residue Asn69* is in contact with the side chain of Leu39. A shift of the side chain amide group of $0.4 \AA$ relative to the wild type enzyme structure is observed (Figure 3). The contacts between Leu39 and residues Asn69* and Tyr 70* enhance interactions between the small domain of one subunit and the large domain of its neighbour, thereby stabilizing the closed conformation. Further differences only occur in the mean temperature factor of the Ile 37 side-chain atoms, which was determined to be $28.9 \AA^{2}$ in the mutant and $37.6 \AA^{2}$ in the wild type enzyme. [Due to the limited resolution of the X-ray diffraction data, temperature factors of the V39Lmaleate complex crystallized from PEG were not treated independently but optimized using the option BGROUP ('grouped temperature factors') in the X-PLOR program package.] The mean overall temperature factor for the two crystal structures of the V39L mutant is $27.9 \dot{\mathrm{A}}^{2}$, whereas the closed structure of the wild type enzyme-maleate complex has a mean $B$-value of $33.1 \AA^{2}$. The difference in the side chain temperature factor indicates reduced flexibility of Ile 37 in the V39L-AS structure, i.e. a stabilization of residues forming the hydrophobic lid on the active site entrance.

Interestingly, the V39L eAspAT crystal form obtained from 
ammonium sulfate is found to be in the closed conformation with a maleate ion bound to the substrate binding pocket. By contrast, in co-crystallization experiments using identical conditions as for $\mathrm{V} 39 \mathrm{~L}-\mathrm{AS}$ the wild type enzyme adopts the half-open conformation. In the wild type structure, the position of the maleate carboxyl group interacting with Arg386 is occupied instead by a sulfate ion (Figure 4 ) which is present in the crystallization buffer approaching a concentration of $1.7 \mathrm{M}$. This provides evidence that the mutant enzyme has a higher affinity, i.e. a lower $K_{\mathrm{d}}$, for maleate than wild type eAspAT. Sulfate, although a competitive inhibitor for AspATs, is not able to trigger the domain movement. These findings support the notion that the mutation of Val39 to Leu causes a shift in the equilibrium from the open towards the closed conformation. The positions of the side chain atoms of residues $69 *$ and 263 are slightly altered with respect to the wild type enzyme structure due to a small shift in the backbone of residues $37-39$. Upon domain opening the side chain of Ile37 is rotated by $\sim 110^{\circ}$ around an axis through the $C_{\alpha}$ atoms of residues $36-38$ (Figure 4), where it snugly fits in a pocket formed by Asn34, Leu35, Asn388 and Gly391 (not shown).

To summarize, the mutant V39L has been crystallized under three different conditions. In all three cases the closed conformation of the enzyme was stabilized. With ammonium sulfate as the precipitant (which does not trigger domain closure for wild type eAspAT), $30 \mathrm{mM}$ maleate could successfully compete with sulfate ions in more than 30 -fold excess for binding in the active site and, thus, cause domain closure. In the presence of only $1 \mathrm{mM} 2$-oxoglutarate the closed conformation was also stabilized. This behaviour of the V39L mutant is in striking contrast to that of wild type eAspAT. The structural differences between the wild type and mutant closed structures are minimal and limited to the immediate environment of residue 39, while maleate binds in exactly the same manner to both active sites (Figure 4). Therefore, the only explanation that the present structures offer for the improved catalytic properties of the V39L mutant is the shift in the open-closed conformational equilibrium. This causes decreases in $K_{\mathrm{m}}$ values of factors between two and ten for five out of six substrates measured (Köhler et al., 1994). Only oxaloacetate does not change its already very small $K_{\mathrm{m}}$ value of $0.02 \mathrm{mM}$ in the mutant enzyme, perhaps because it reacts so avidly with the co-factor PMP that domain closure is irrelevant to the process of binding (Picot et al., 1991). This effect of the mutation V39L is confirmed by the opposite behaviour of the L39V mutant of eTyrAT, which results in increased $K_{\mathrm{m}}$ values (and slightly reduced $k_{\text {cat }}$ values) compared with wild type eTyrAT (Köhler et al., 1994).

A comparison of the properties of wild type and V39L eAspAT nicely illustrates the subtle structural features that determine the enzyme's dynamic behaviour. It does not, however, explain the difference in specificity between eAspAT and eTyrAT. The weak binding of aromatic substrates to eAspATs [saturation of the active site was not observed in eAspAT for Tyr and Phe, for example (Hayashi et al., 1991; Köhler et al., 1994)] clearly necessitates a significant further stabilization of the closed structure in order to obtain $K_{\mathrm{m}}$ values for aromatic substrates $<0.1 \mathrm{mM}$ (Hayashi et al., 1993). However, there also have to be mutations that are favourable only for aromatic amino and keto acid substrates. That the effects of point mutations are not necessarily additive, as e.g. double and triple mutants of eAspAT studied by Köhler et al. (1994) demonstrate, is a complication. Attempts to obtain multiple eAspAT mutants with properties more similar to those of eTyrAT are nonetheless continuing in the laboratory of K.Kirschner and, independently, in that of J.F.Kirsch (UC, Berkeley).

\section{Acknowledgements}

We are grateful to E.Kohler and K.Kirschner for providıng the mutant enzyme and results of the biochemical characterization. The overproducing $E$.coli strain and the plasmid containing the asp $C$ gene were kindly supplied by I.Fotheringham and M.Edwards. We gratefully acknowledge Dr P.Tucker for the data collection on the XENTRONICS area detector at EMBL, Heidelberg, Germany. We thank Drs P.F Jones, M D.Toney and S.J.Smerdon for helpful discussions and critical reading of the manuscript. This work was supported by grants from the Swiss National Science Foundation (3.098-0.85 and 31.25713.88 to J.N.J.).

\section{References}

Amone,A., Christen,P., Jansonius,J.N, and Metzler,D.E. (1985a) In Christen,P. and Metzler,D.E. (eds), Transaminases. John Wiley \& Sons, New York, pp. 326-362.

Arnone,A., Rogers,P.H , Hyde,C.C., Briley,P.D., Metzler,C.M. and Metzler,D.E. (1985b) In Christen,P. and Metzler,D.E. (eds), Transaminases. John Wiley \& Sons, New York, pp. 138-155.

Borisov,V.V., Borisova,S.N., Kachalova,G.S., Sosfenov,N.I. and Vainshtein,B.K. (1985) In Christen,P. and Metzler,D.E. (eds), Transaminases. John Wiley \& Sons, New York, pp. 155-164.

Braunstein,A.E. (1973) In Boyer,P.D. (ed.), The Enzymes. 3rd edn, Vol. 9. Academic Press, New York, pp. 379-481.

Christen,P. and Metzler,D.E. (eds) (1985) Transaminases. John Wiley \& Sons, New York.

Cleland,W.W. (1963) Biochim. Biophys. Acta, 67, 104-137.

Cronin,C.N. and Kirsch,J.F. (1988) Blochemistry, 27, $4572-4579$.

Crowther,R.A. (1972) In Rossmann,M.G. (ed.), The Molecular Replacement Method. Gordon and Breach, New York, pp. 173-178.

Crowther,R.A. and Blow,D.M. (1967) Acta Crystallogr., 23, 544-548.

Doonan,S. et al. (1975) Biochem, J., 149, 497-506.

Eichele,G., Ford,G.C., Glor,M., Jansonius,J.N., Mavrides,C. and Christen,P. (1979) J. Mol. Biol., 133, 161-180.

Fersht,A. (1985) Enzyme Structure and Mechanism. W.H.Freeman, New York, pp. $105-106$

Ford,G.C., Eichele,G. and Jansonius,J.N. (1980) Proc. Natl Acad. Sci. USA, 77, 2559-2563

Fotheringham,I., Dacey,S., Taylor,P., Smith,T., Hunter,M., Finlay,M., Primrose,S., Parker,D. and Edwards,M. (1986) Biochem. J., 234, 593-604.

Hayashi,H., Kuramitsu,S. and Kagamiyama,H. (1991) J. Biochem. (Tokyo), 109, $699-704$

Hayashı,H., Inoue,K., Nagata,T., Kuramitsu,S. and Kagamiyama,H. (1993) Biochemistry, 32, $12229-12239$.

Howard,A.J., Gilliland,G.L., Finzel,B.C., Poulos,T.L., Ohlendorf,D.H. and Salemme,F.R. (1987) J. Appl. Crystallogr., 20, 383-387.

Inoue, Y., Kuramitsu,S., Inoue,K., Kagamiyama,H., Hiromi,K., Tanase,S. and Morno,Y. (1989) J. Biol. Chem., 264, 9673-9681.

Jäger,J. (1991) Ph.D. thesis, University of Basel.

Jager,J. et al. (1989) J. Mol. Biol., 209, 499-501.

Jäger,J., Solmajer,T. and Jansonius,J.N. (1992) FEBS Lett., 306, 234- 238.

Jäger,J., Moser,M., Sauder,U. and Jansonius,J.N. (1994) J. Mol. Biol., in press.

Jansonius,J.N. and Vincent,M.G. (1987) In Jurnak,F.A. and McPherson,A. (eds), Biological Macromolecules and Assemblies. Vol. 3: Active Sites of Enzymes. John Wiley \& Sons, New York, pp. 187-285.

Jansonius,J.N., Eichele,G., Ford,G.C., Picot,D., Thaller,C. and Vincent,M.G (1985) In Christen,P. and Metzler,D.E. (eds), Transaminases. John Wiley \& Sons, New York, pp. 110-138.

Jenkins,W.T., Yphantis,D.A. and Sizer,I.W. (1959) J. Biol. Chem., 234, 51-57. Jones, T.A. (1985) Methods Enzymol, 115, 157-171.

Kamitori,S. et al. (1987) J. Biochem. (Tokyo), 101, 813-816.

Kamitori,S. et al. (1988) J. Biochem. (Tokyo), 104, 317-318.

Kamitori,S., Okamoto,A., Hirotsu,K., Higuchi,T., Kuramitsu,S., Kagamiyama,H., Matsuura,Y. and Katsube, Y. (1990) J. Biochem. (Tokyo), 108, $175-184$.

Kirsch,J.F., Eichele,G., Ford,G.C., Vincent,M.G., Jansonius,J.N., Gehring,H. and Christen,P. (1984) J. Mol. Biol., 174, 497-525.

Köhler,E. (1990) Ph.D. thesis, University of Basel.

Köhler,E., Seville,M., Jäger,J., Fotheringham,I., Hunter,M., Edwards, M., Jansonius,J.N. and Kirschner,K. (1994) Biochemistry, 33, 90-97.

Kraulis,P.J. (1991) J. Appl. Crystallogr., 24, 946-950.

Kuramitsu,S., Inoue, Y., Tanase,S., Morino,Y. and Kagamiyama,H. (1987) Biochem. Biophys. Res. Commun., 146, 416-421. 


\section{J.Jäger $e t$ al.}

Kuramitsu,S., Hiromi,K., Hayashi,H., Morino,Y. and Kagamiyama,H. (1990) Biochemistry, 29, 5469-5476.

Luzzati,V. (1952) Acta Crystallogr., 5, 802-810.

McPherson,A. (1982) Preparation and Analysis of Protein Crystals. John Wiley \& Sons, New York, pp. 94-96.

Malcolm,B.A. and Kirsch,J.F. (1985) Biochem. Biophys. Res. Commun., 132, 915-921.

Matthews,B.W. (1968) J. Mol. Biol., 33, 491-497.

Mavrides, C. and Christen,P. (1978) Biochem. Biophys. Res. Commun., 85, $769-773$.

Mehta,P.K., Hale,T.I. and Christen,P. (1989) Eur. J. Biochem., 186, 249-253.

Messerschmidt,A. and Pflugrath,J.W. (1987) J. Appl. Crystallogr., 20, 306-315.

Ovchinnikov, Yu.A. et al. (1973) FEBS Lett., 29, 31-34.

Picot,D., Sandmeier,E., Thaller,C., Vincent,M.G., Christen,P and Jansonius,J.N. (1991) Eur. J. Biochem., 196, 329-341.

Powell,J.T. and Morrison,J.F. (1978) Eur. J. Biochem., 87, 391-400

Read,R.J. (1986) Acta Crystallogr., A42, 140-149.

Seville,M., Vincent,M.G. and Hahn,K. (1988) Biochemistry, 27, 8344-8349.

Shrawder,E. and Marunez-Carrion,M. (1972) J. Biol. Chem., 247, 2486-2492.

Smith,D.L., Ringe,D., Finlayson,W L. and Kirsch,J.F. (1986) J. Mol. Biol., $191,301-302$.

Smith,D.L., Almo,S.C., Toney,M.D. and Ringe,D. (1989) Biochemistry, 28. $8161-8167$.

Sussman,J.L., Holbrook,S.R., Church,G.M. and Kım,S. (1977) Acta Crystallogr., A33, 800-804.

Tickle,I.J. (1985) In Machin,P.A. (ed.), Molecular Replacement. Science and Engineering Research Council, Daresbury, pp. 22-26.

Toney,M.D. and Kırsch,J.F. (1987) J. Biol. Chem., 262, 12403-12405.

Torchinsky,Y.M. (1986) In Dolphin,D., Poulson,A. and Avramović,O. (eds), Vitamm B6, Pyridoxal Phosphate. Part B. John Wiley \& Sons, New York, pp. $169-221$.

Tronnud,D.E., Ten Eyck,L.F. and Matthews,B.W. (1987) Acta Crystallogr., A43, 489-501.

Received September 20, 1993; accepted February 21, 1994 